\title{
An Investigation on Stability and Operability in Haptic Communication Systems
}

\author{
Hitoshi Watanabe ${ }^{1+}$, Pingguo Huang ${ }^{2}$ and Yutaka Ishibashi ${ }^{3}$ \\ ${ }^{1}$ Faculty of Engineering, Tokyo University of Science, Tokyo, Japan \\ ${ }^{2}$ Faculty of Business Management, Seijoh University, Tokai, Japan \\ ${ }^{3}$ Graduate School of Engineering, Nagoya Institute of Technology, Nagoya, Japan
}

\begin{abstract}
In the remote robot systems using haptic communication under communication delays, there is a problem of instability of operations or behaviors such as mechanical howling. For solving the problem, we have introduced a method using "resistance" which is proportion to the velocity and investigated the theoretical method to analyze the mechanism by using difference differential equations. In our previous theoretical research, we have clarified that the resistance has the effect to decrease the mechanical howling but it degrades the operability of the action to approach to the target position. These results suggest that the minimum resistance which does not cause the mechanical howling is the optimal resistance value which keeps the operability of the target approaching action high as much as possible. This study investigates the feasibility of this method by experiment and theoretical analysis.
\end{abstract}

Keywords: Haptic communication; Communication delay; Stability; Operability; Resistance; Difference differential equation; Human characteristics

\section{Introduction}

The haptic communication attracts great attention now [1] and applications for many fields, e.g. telesurgery, are being investigated [2]. However, when a remote robot system with haptics is executed through a network in which the QoS (Quality of Service) [3] is not guaranteed like as the Internet, there is the possibility that the QoE (Quality of Experience) [4] may degrade heavily. For solving this problem, we have introduced a method using "resistance" [5] which is proportion to the velocity and investigated the effects of this method by experiment using real systems. Moreover, we have investigated the theoretical analysis [6], [7], [8] to clarify the mechanism by using difference differential equation [9], [10]. In our previous theoretical research, we have clarified that the resistance has the effect to decrease the mechanical howling but it degrades the operability of the action to approach the target position [11]. Moreover, we have also investigated the basic frame to determine the optimal resistance based on the estimation of human operation characteristics by experiment [8]. This paper investigates the feasibility of the method to keep the operability high as much as possible and suppress the mechanical howling by choosing the optimal value of the resistance.

The new theoretical consideration is added to the previous research and experimental trial to clarify the feasibility of the proposed method has been done by using the haptic communication system.

\section{The Behavior of Remote Robot Systems}

Fig. 1 shows the behaviour of the remote robot systems. The moving of the object is executed by determining the position at the next time by the master's instruction. The reaction force which is required for 
moving the object under the certain kinetic law is returned to the master as the "reaction force". Here, $S_{t}$ is the position of slave at time $t, M_{t}$ is the instructed position by the maser at time $\mathrm{t}$ and $F_{t}$ is the reaction force.

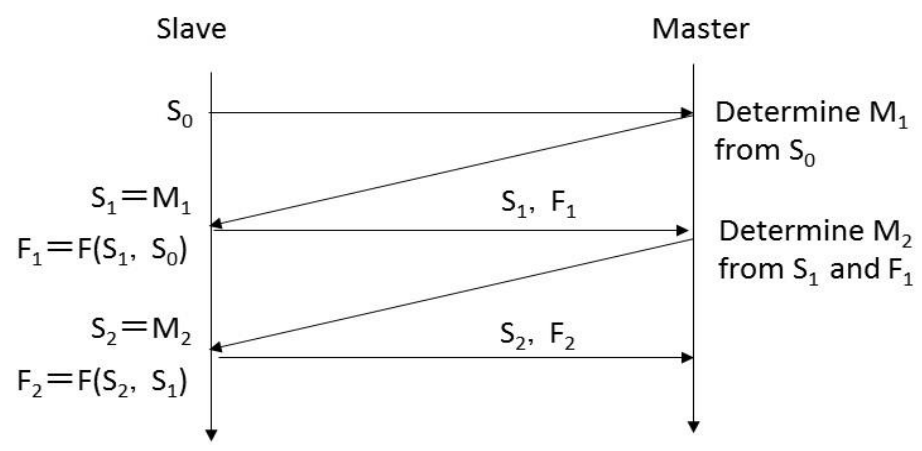

Fig.1: Behaviour of Remote Robot System

The resistance which we introduced is the following coefficient $C_{d}$ converting the $M_{t}$ to the $S_{t}$.

$$
\begin{array}{r}
S_{\mathrm{t}_{+}+}=M_{\mathrm{t}}-C d(M t-S t) \\
0 \leq C d<1
\end{array}
$$

The operator manipulates as to approach the object to the target position. This action is done by considering the present position of the object and the reaction force. This is the feedback loop with time delay. On the other hand, for the feedback of reaction force from slave to master, it is necessary to transform the reaction force to some displacement at the master. One of its methods is to use the spring-dumper mechanism. This is another feedback loop with time delay.

\section{The Feedback Loop for Target Approaching Action}

Let $y(t)$ be the position of the object at time $t, V$ be the velocity of manipulation for approaching to the target position and, $y_{D}$ be the target position. Also $V$ is assumed as a function of $y(t)-y_{D}$ as formula (2) [6].

$$
V(t)=V\left(y(t)-y_{D}\right)
$$

$V$ is the velocity of the manipulating position but not match to the velocity of the position of the object, i.e., in the case where the resistance $C_{d}$ is introduced. Furthermore, in the case where the communication delay is $\delta, V$ is determined based on the position of object time $\delta$ ago. Therefore, the following formula is obtained.

$$
\frac{d y(t)}{d t}=F\left(V\left(y(t-\delta)-y_{D}\right)\right)
$$

Here, $F$ is the function which associates the position of the master and one of the slaves. Formula (3) is a difference differential equation. Assuming that the position of the master matches to the one of the slaves and the velocity function has formula (4), the behaviour of the solution depends $\alpha$ and $\delta$. An example is shown in Fig 2.

$$
V(y)=A(y-y d)^{\alpha}
$$

Whether the solution vibrates or oscillates or not is the important point to evaluate the stability and operability. It is decided by the examination of the characteristic function, if $V$ is a linear function. Let $y(t)=C e^{-\lambda t}$ be a solution of the following formula (5).

$$
\frac{d y(t)}{d t}=-A y(t-\delta)
$$

$\lambda$ should satisfy formula (6).

$$
\begin{aligned}
\lambda \delta & +A \delta e^{-\lambda \delta}=0 \\
\therefore \quad & u+k e^{-u}=0
\end{aligned}
$$


Here, $\mathrm{k}=\delta / A$. Formula (6) is called the characteristic equation. Examining the positive or negative of the real part of the roots of formula (6), the stability of the solution of formula (5) can be decided [10]. For example, the solution does not vibrate and converge to 0 when $\delta<(1 / e)(1 / A)$, and the solution vibrates and diverges to infinity when $\delta>\pi /$ A (see Fig.3).

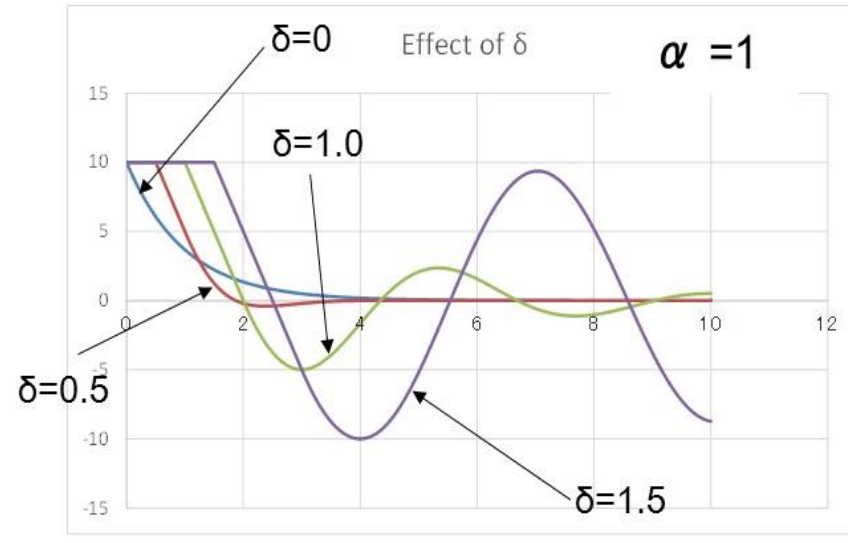

(a) Effect of communication Delay

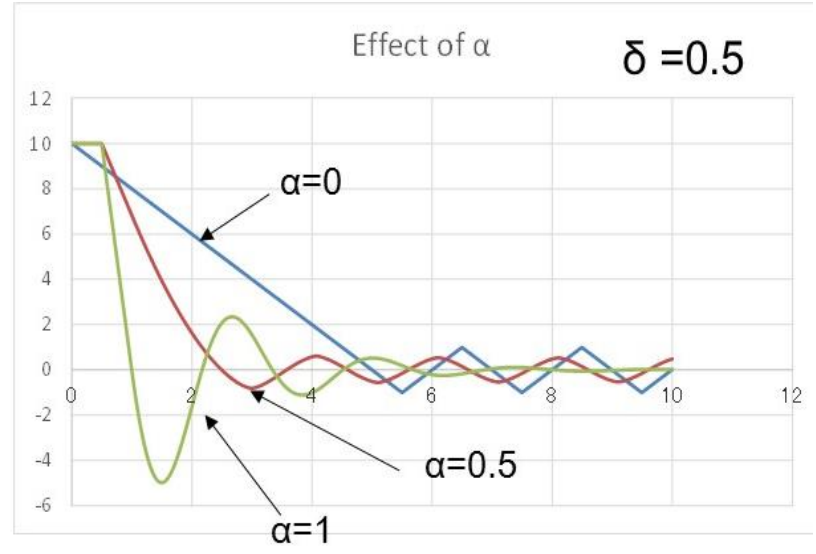

(b) Effect of function $\mathrm{V}$

Fig. 2: Effect of communication delay and velocity function

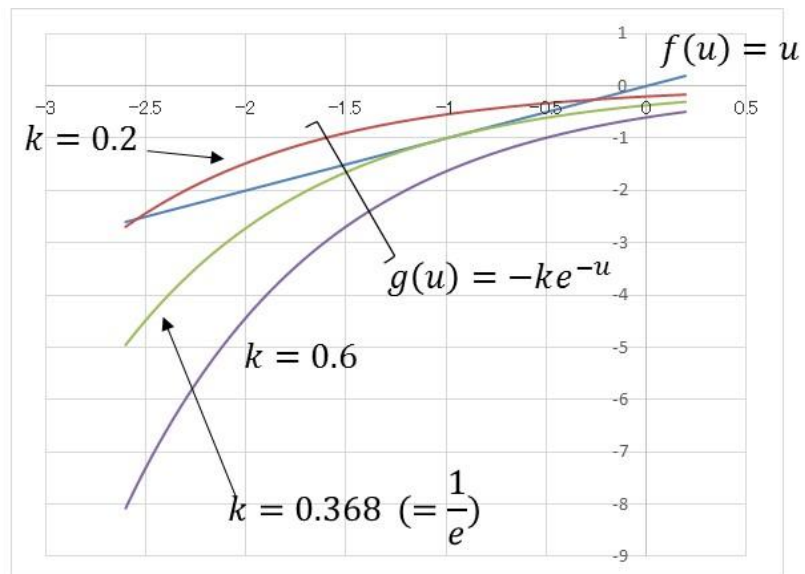

The real roots of equation (6)

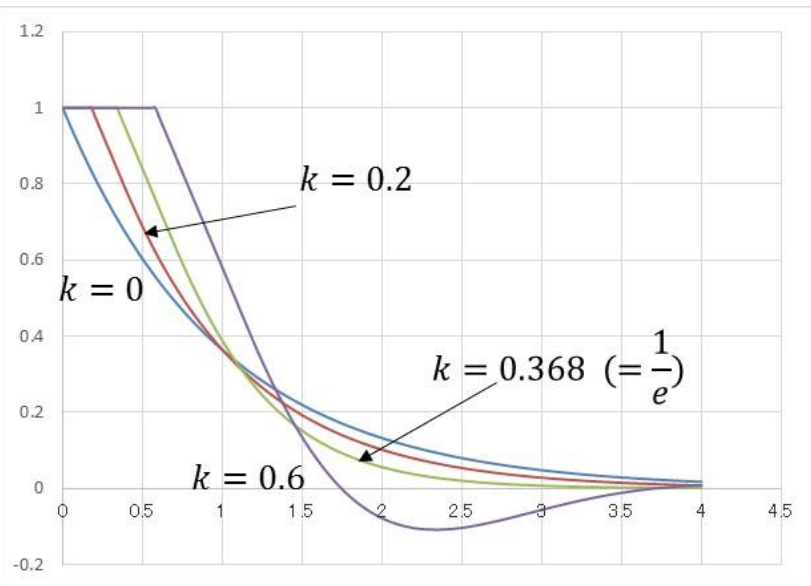

The solution of the equation (5)

Fig. 3: The behaviour of the solution of (5)

We have applied this method for evaluating the stability when the resistance $C_{d}$ is introduced. When the control period of the remote robot system is adequately small, the input function $g(t)$ is converted to the output function $f(t)$ by formula (7) [7]. It means that $C_{d}$ is a kind of the first-order delay element. Here, $k=\Delta$ $\left(1-C_{d}\right) / C_{d}$ and $\Delta$ is the sampling period. The difference differential equation is obtained as formula (8). The characteristic equation is obtained as formula (9).

$$
\begin{gathered}
f(t)=k e^{-k t} \int_{-\infty}^{t} e^{k u} g(u) d u \\
\tau \frac{d 2 f(t)}{d t 2}+\frac{d f(t)}{d t}+A f(t-\delta)=0 \\
\text { here } \tau=\frac{1}{v}=\frac{\Delta C d}{1-C d} \\
\frac{\tau}{\delta} u^{2}+u+k e^{-u}=0
\end{gathered}
$$

The range of communication delay which gives the real root of the characteristic equation of formula (9) is narrower than the case of no $C_{d}$ (Fig. 4(a)). It means that the $C_{d}$ is an instability factor to the target approaching action (Fig.4 (b)). 


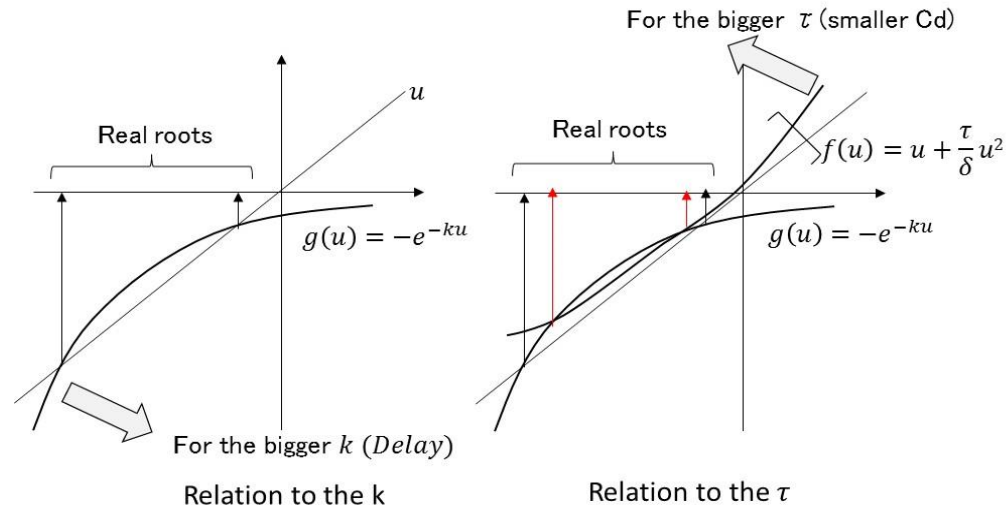

(a) The existence condition of real roots

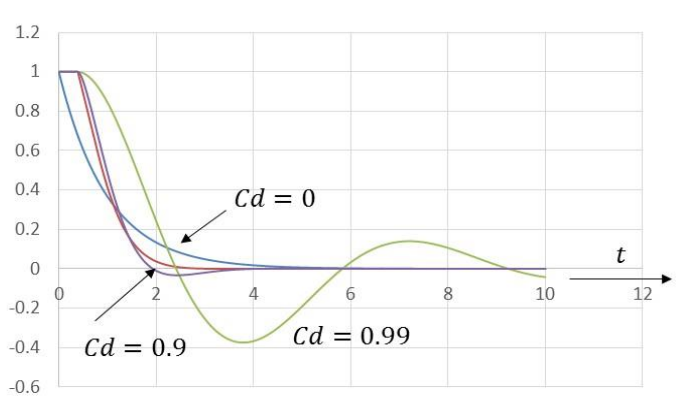

(b) Transient solutions for some Cds

Fig. 4: The behaviour of solution of (5)

\section{The Effect to the Reaction Force Feedback}

For the feedback of reaction force from slave to master, it is necessary to transform the reaction force to some displacement at the master. The representative means is to use the spring dumper mechanism in the master's interface. In this case, the feedback loop of the reaction force is described as the control brock diagram as shown in Fig. 5 [12]. The Equation of motion of the system shown in Fig. 5 with communication delay $\delta$ is described as formula (10).

$$
\frac{m}{c} \frac{d^{2} y(t)}{d t^{2}}+\frac{d y(t)}{d t}+\frac{\kappa}{c} y(t-\delta)=0
$$

where $m$ is the mass, $\kappa$ is the spring constant and $c$ is the dumper constant.

Introducing the resistance to this system, the difference differential equation and the characteristic equation are obtained as formulae (11) and (12).

$$
\begin{gathered}
m \frac{d^{3} y(t)}{d t^{3}}+\left(c+\frac{m}{\tau}\right) \frac{d^{2} y(t)}{d t^{2}}+\frac{c}{\tau} \frac{d y(t)}{d t}+\frac{\kappa}{\tau} y(t-\delta)=0 \\
m \frac{d^{3} y(t)}{d t^{3}}+\left(c+\frac{m}{\tau}\right) \frac{d^{2} y(t)}{d t^{2}}+\frac{c}{\tau} \frac{d y(t)}{d t}+\frac{\kappa}{\tau} y(t-\delta)=0
\end{gathered}
$$

Introducing big $\mathrm{C}_{\mathrm{d}}$ means big $\tau$. Examing the characteristic equation (12), it is concluded that the bigger $C_{d}$ contributes to the system stability (Fig.6).

\section{Towards the Optimization of $C_{d}$}

From the above theoretical analysis, the resistance $C_{d}$ has the effect to decreasing mechanical howling but it is the degrading factor to the target approaching action. Therefore, the one of the methods to optimize the value of $C_{d}$ is to determine as the minimum value not to cause the mechanical howling. To estimate the system parameters from the experiment and to obtain the minimum $C_{d}$ from the examination of the characteristic equation is a possible way. To say concretely, the stability condition is that the characteristic equation has only real roots.

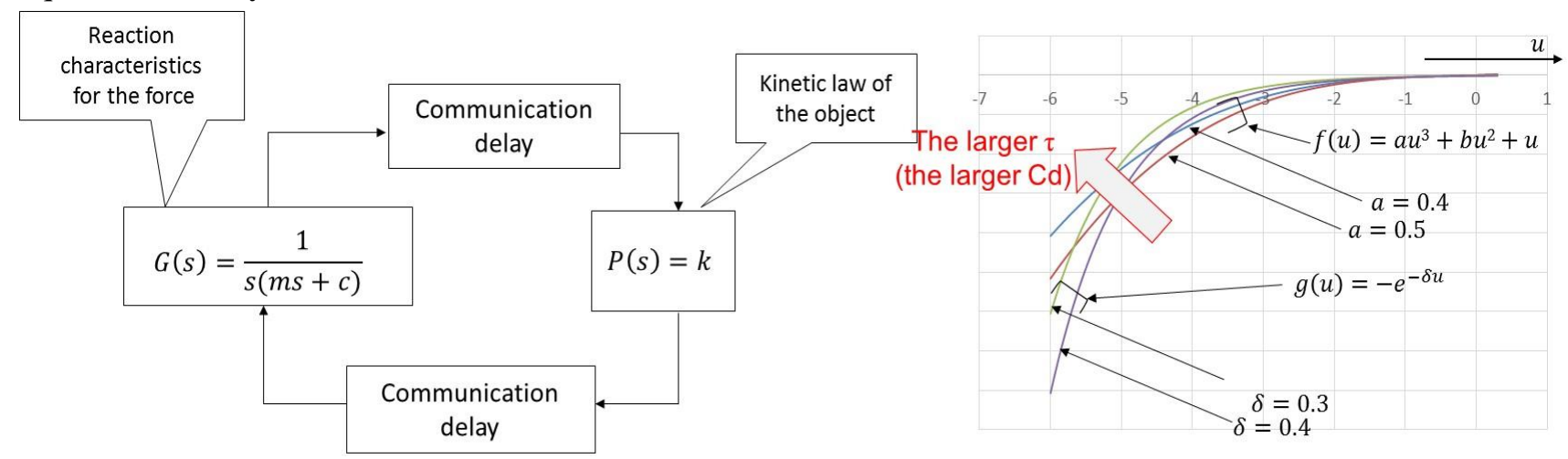

Fig. 5: The brock diagram of the force feedback

Fig. 6: Examination of the real roots of equation (12) 
Although these analytical methods are imagined, firstly it is necessary to understand human characteristics. There are two human characteristics according to the two feedback loops. Of course the target approaching action depends on the human characteristics, but also the force feedback depends on the human characteristics. Because the element of kinetic parameter such as the dumper parameter contains the operator's physical characteristics.

\section{Human Characteristics of Target Approaching Action}

The target approaching human characteristics is returned to the function described in formula (2). We have tried to grasp human characteristics by experiment using the distributed game system with haptic communication [13], [8]. The system is a game in which players move an object on a virtual space. The objects are moved along the maze and the maze can be constructed by using the movable walls. The players move the objects to the settled goal (Fig. 7). The communication delay can be changed.

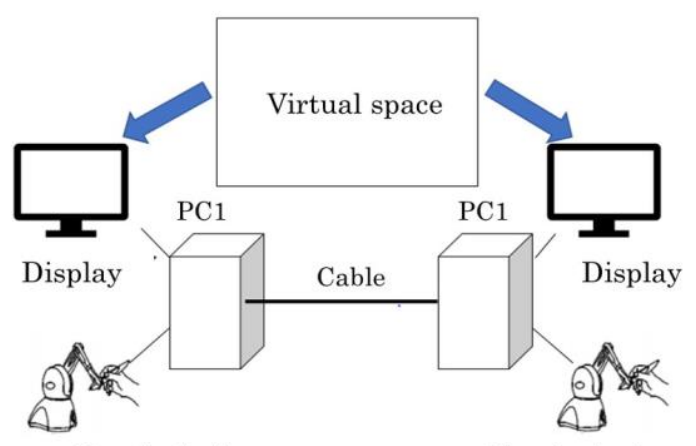

Haptic device

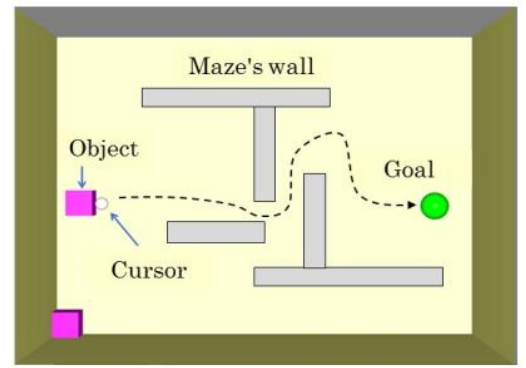

The virtual space consists of objects, cursors, walls and the goal.

Fig. 7: System configuration of developed game

By using the system, the following analysis becomes to be possible. (a) Play not only in the simple route, but also in various complex routes. (b) Not only the standalone work, but also the competitive or cooperative work can be analysed. (c) The effect of communication delay can be investigated.

The obtained data from this system is the chronological ordered data of time and position $(t, y(t))$. The parameter $\mathrm{A}$ and $\alpha$ is estimated by this data. For estimating these parameters from the data, it is thought to be expressed as the scatter plot of $(y, V)$. However, the destination point exists only in the operator's head and cannot be measured from outside data. Therefore, we have proposed the following method. The derivation of formula (3) under the assumption that A and $\alpha$ are constant yields formula (13).

$$
\log \left(\frac{d^{2} y(t)}{d t^{2}}\right)-\log \left(\frac{d y(t-\delta)}{d t}\right)=\left(\frac{1}{\alpha}\right) \log A+\log \alpha+\left(1-\frac{1}{\alpha}\right) \log \frac{d y(t)}{d t}
$$

Formula (13) means that $\ln \left(d^{2} y / d t^{2}\right)$ is a linear function of $\ln (d y / d t)$. Therefore, from the linear regression of the scattered plot of $\ln \left(d^{2} y / d t^{2}\right)$ and $\left.\ln (d y / d t)\right), A$ and $\alpha$ can be obtained. The followings are the estimation example. Fig. 8(a) is the moving route of the object, Fig. 8(b) is the relationship of position and velocity, and Fig. 8(c) and Fig. 8(d) are the estimation results of $A$ and $\alpha$ for different delay conditions.

The velocity functions in Fig. 8(c) and (d) are different from each other. The theoretical analysis using the estimated parameter under no delay says that the actual action has smaller vibration than predicted one by theoretical analysis. This is thought that the operator might absorb the effect of communication delay unconsciously and it is appeared to the difference of velocity function. Therefore, the difference of velocity function might be a large factor of QoE of the haptic systems.

\section{Experiment of the Mechanical Howling}

The human characteristics related to the mechanical howling has been analysed by experiment. Moreover, the estimation method of the velocity function has also been improved.

\subsection{Improvement of the Estimation Method}

The observed positon $y(t)$ is different form the master's instructed position if the $C_{d}$ is introduced. Let $m(t)$ be the master's instructed position. In this case, the velocity function is $d m(t) / d t$ in formula (14). 


$$
\frac{d m(t)}{d t}=-A\left(y(t-\delta)-y_{D}\right)^{\alpha}
$$

Firstly, get $m(t)$ from $y(t)$ by using formula (1) and analyse the chronological ordered data of time and position $(t, y(t))$. The deviation of formula (14) yields the following formula.

$$
\log \left(\frac{d^{2} \boldsymbol{m}(t)}{d t^{2}}\right)-\log \left(\frac{d y(t)}{d t}\right)=\left(\frac{1}{\alpha}\right) \log A+\log \alpha+\left(1-\frac{1}{\alpha}\right) \log \frac{d m(t)}{d t}
$$

After here, the estimation method is same as mentioned in section 6. However, estimation is not done when the contribution rate in the current window is less than the certain value. In such a case, the velocity changes with rapidly like vibration.

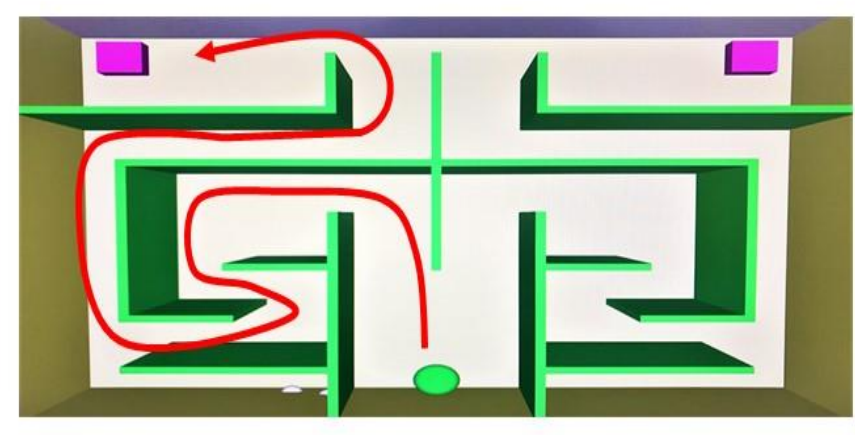

(a) Moving route

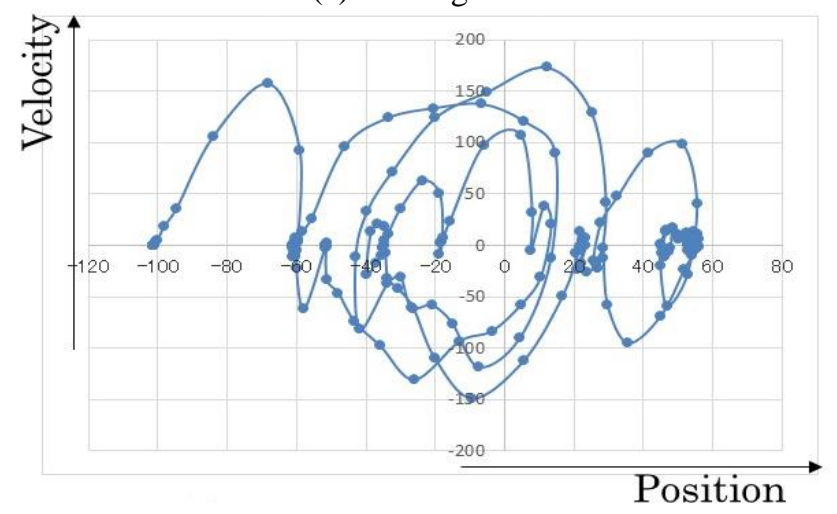

(b) Position and velocity

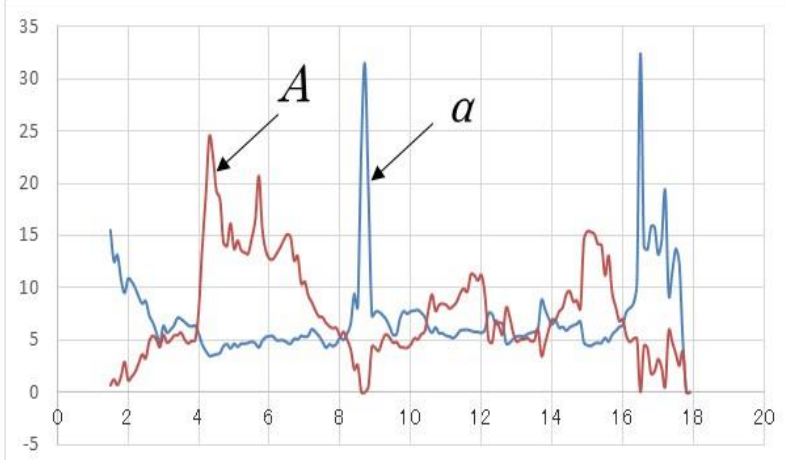

(c) Velocity in on delay

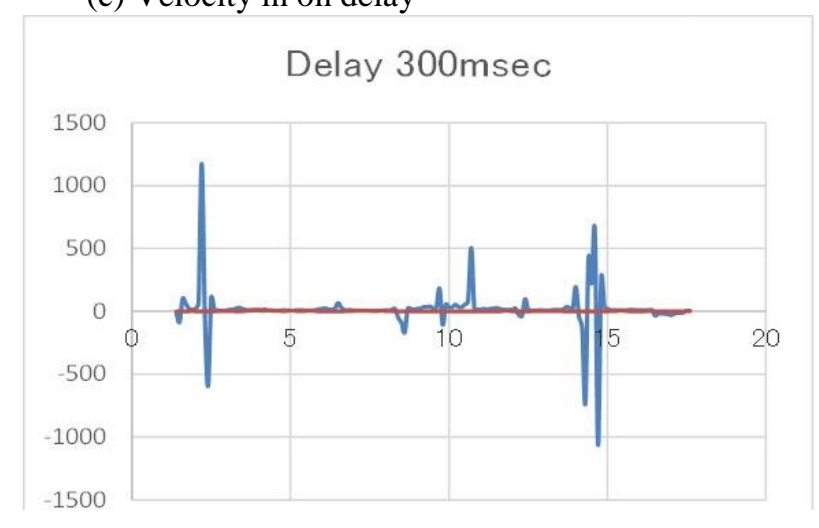

(d) Velocity in communication delay

Fig. 8: Estimation example of velocity function

\subsection{Estimation Examples}

The experiment is carried out by the route shown in Fig. 9. Object is moved from the start point and pass the narrow passage and hit against the wall. Fig. 10 is an example of the 2-dimension trajectory. The part circled in the figure means the cause of mechanical howling. With or without of mechanical howling under some condition of $C_{d}$ and communication delay are shown in Fig.11. When $C_{d}$ is large the mechanical howling is suppressed even the case of large communication delay. This is consistent to our theory.

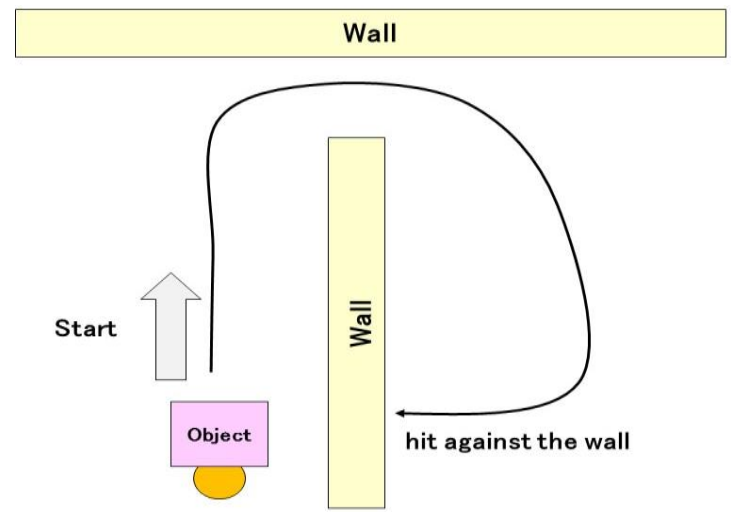

Fig. 9: The experiment route

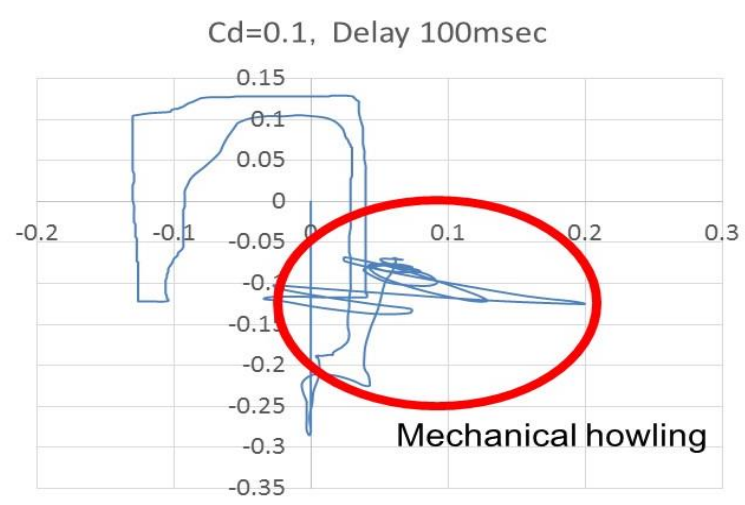

Fig. 10: A example of trajectory 


\begin{tabular}{|c|c|c|c|c|c|}
\hline \multirow{2}{*}{ Cd } & \multicolumn{5}{|c|}{ Delay (msec) } \\
\cline { 2 - 6 } & 0 & 50 & 100 & 200 & 300 \\
\hline 0.1 & Severe & Slightly & Severe & Slightly & Slightly \\
\hline 0.3 & No & - & - & - & Slightly \\
\hline 0.5 & Slightly & - & - & - & Severe \\
\hline 0.7 & Slightly & - & - & - & No \\
\hline 0.9 & No & No & No & No & Slightly \\
\hline
\end{tabular}

Fig. 11: With or without of mechanical howling

Fig. 12 shows the temporal change of position and velocity function of a specific player. The temporal change of position is greatly different according to the condition of $C d$ and communication delay. However, the velocity function hardly change. This is different from the result mentioned in section 6 . It is thought that the work condition might affect to the player's operation. The maze of this experiment is not complex as section 6. The results in former section shows that the solution of difference differential equation changes according to delay and $C_{d}$ if the velocity function is same. Therefore, the change of positon is great and the change of velocity function is small is consistent to our theory.

Fig. 13 shows the individual differences of velocity function under similar condition. The individual differences are large. Player 3 has smaller $\alpha$ than other player. The small $\alpha$ means the rapid acceleration deceleration. This characteristics of player 3 reflects the temporal change of the positon.
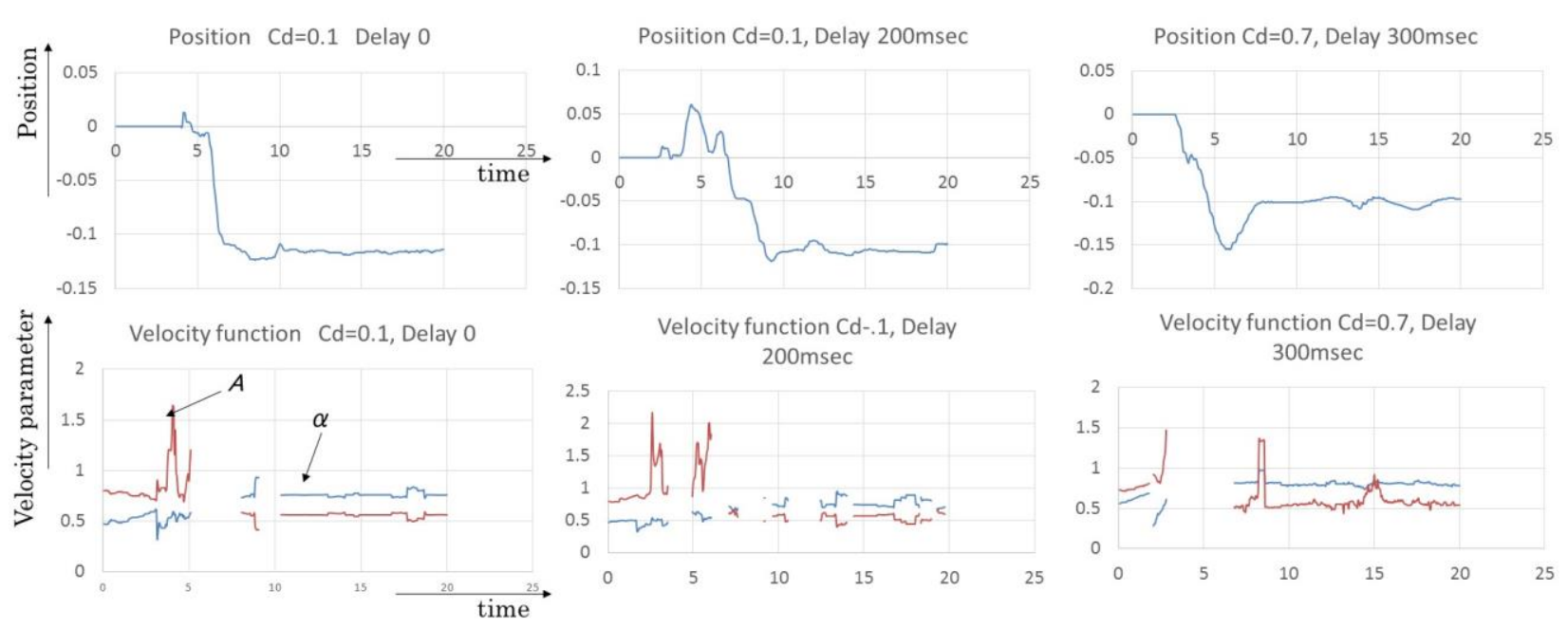

Fig. 12: Change in Velocity Function

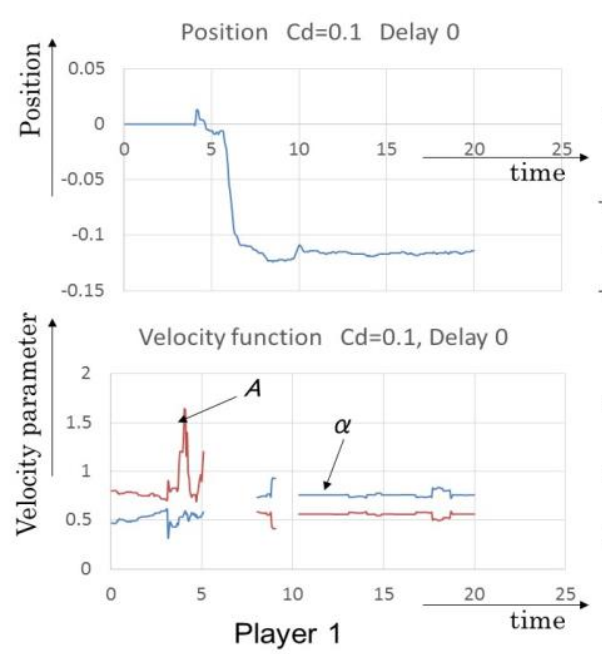

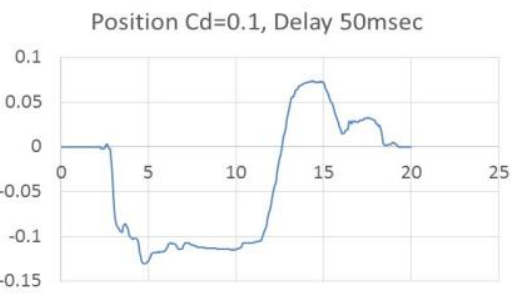

Velocity function $\mathrm{Cd}=0.1$, Delay $50 \mathrm{msec}$

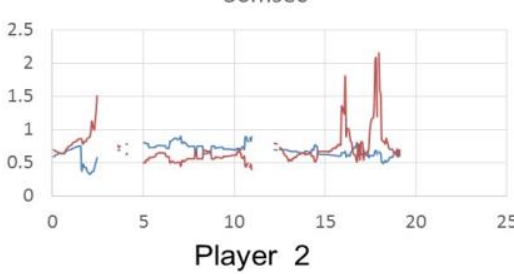

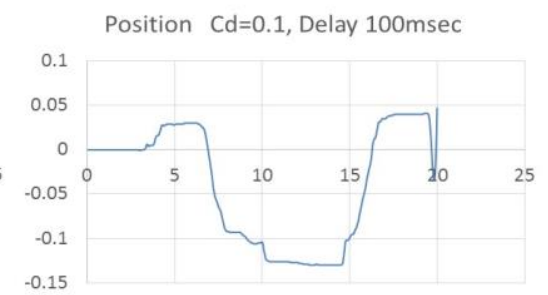

Velocity function $\mathrm{Cd}=0.1$, Delay $100 \mathrm{msec}$

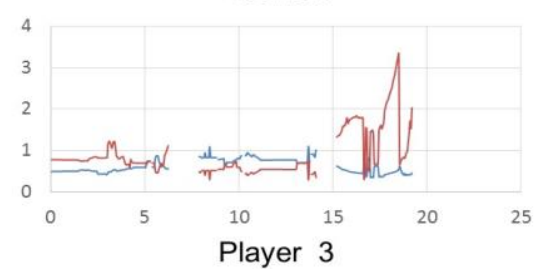

Fig. 13: Individual difference of velocity function 


\section{Conclusion}

This paper investigated the feasibility of the determination of optimum resistance to satisfy both stability and operability. Our previous studies have been summarized and the new results have been presented. From the experiment, including our previous studies, operators adjust their motion under the communication delay to decrease the vibrations. But these phenomena are depend on many conditions such as kind of work, complexity of work and so on. Furthermore, the individual different of velocity function is large. The final purpose of this research is to realize the good operability and the stability. Generally speaking, $\mathrm{Cd}$ is a kind of signal conversion and to find the optimal conversion is the future problem. To solve this problem, the feedback for approaching the target position and the feedback of reaction force should be integrated and more investigation to clarify the operating characteristics is required.

\section{Acknowledgements}

This work was partly supported by JSPS KAKENHI Grant Number 18K11261.

\section{References}

[1] K. Ohnishi, "Real world haptics : Its principle and future prospects," The journal of the Institute of Electrical Engineers of Japan (IEEJ), vol. 133, no. 5, pp.268-269, Mar. 2013.

[2] T. Kawai, "Haptics and surgery," IEEJ Journal, vol. 133, no. 5, pp. 282-285, Mar. 2013.

[3] ITU-T Rec. E. 800, "Terms and definitions related to quality of service and network performance including dependability," 1994.

[4] ITU-T Rec. G. 100/P. 10 Amendment 1. New appendix I - Definition of quality of experience (QoE), 2007

[5] Y. Komatsu, H. Ohnishi, and Y. Ishibashi, "Adaptive control of viscosity in remote control system with force feedback," in Proc. IEEE International Conference on Consumer Electronics - Taiwan (ICCE-TW), pp. 237-238, June 2017.

[6] H. Watanabe, Y. Ishibashi, and P. Huang, "A formulation of remote robot system by using difference differential equation," in Proc. IEEE The 3rd International Conference on Computer and Communication Systems (ICCCS), pp. 511-515, Apr. 2018.

[7] H. Watanabe, P. Huang, and Y. Ishibashi, "An investigation of the stabilization of bilateral robot systems under communication delay," in Proc. IEEE International Conference on Intelligence and Safety for Robotics (ISR), pp. 140-145, Aug. 2018.

[8] H. Watanabe, K. Kuroyanagi, Z. Sato, H. Hirado, P. HUANG and Y. Ishibashi, " A Proposal of the Method for Analyzing the Stability of Virtual Distributed Systems using Haptic Communication," in Proc. IEEE International Conference on Consumer Electronics - Taiwan (ICCE-TW), pp. 55-56, May 2018.

[9] S. Sugiyama, "Difference differential equation," Kyoritsu-shuppan, 1971.

[10] R. Miyazaki, "Introduction to the theory of delay differential equations," Report of the Research Institute for Mathematical Sciences, Kyoto University, published in 2010.

[11] H. Watanabe, Y. Ishibashi, and P. Huang," A Stability Analysis of Haptic Systems by Using Difference Differential Equation, -From the view point of characteristic equation- " ,in Proc. The 2nd World Symposium on Communication Engineering (WSCE 2019), pp. 127-132, Dec. 2019.

[12] T. Miyoshi, "Stabilized Control in Haptic Communications", J. IEICE, Vol.102 No.1 pp.57-63, 2019.

[13] Pingguo Huang, Yutaka Ishibashi, "QoE assessment of will transmission using vision and haptics in networked virtual environment", International Journal of Communications, Network and System Sciences (IJCNS), vol. 7, no. 8, pp. 265-278, Aug. 2014.) 